\title{
NUEVOS REGISTROS DE PECES PARA EL ÁREA MARINA DEL PARQUE NACIONAL SISTEMA ARRECIFAL VERACRUZANO, SUROESTE DEL GOLFO DE MÉXICO
}

\author{
New fishes records in the marine area from \\ Veracruz Reef System National Park, Southwestern Gulf of Mexico
}

\begin{abstract}
Luis Fernando Del Moral-Flores ${ }^{1 \mathrm{a},}$, , Eduardo López-Segovia ${ }^{1,2}$ y Tao Hernández-Arellano ${ }^{1 b}$
${ }^{1}$ Laboratorio de Zoología, Facultad de Estudios Superiores Iztacala, Universidad Nacional Autónoma de México (UNAM), Av. de los Barrios No. 1, Los Reyes Iztacala, 54090 Tlalnepantla, Estado de México, México; (1D orcid.org/0000-0002-7804-2716; 1b (1) orcid.org/0000-0003-0898-5978. ${ }^{2}$ Posgrado en Ciencias del Mar y Limnología, Universidad Nacional Autónoma de México; Av. Ciudad Universitaria 3000, C.P. 04510, Coyoacán, Ciudad de México, México; (1) orcid.org/0000-0001-8065-1521. "Para correspondencia: delmoralfer@gmail.com.
\end{abstract}

\section{RESUMEN}

Presentamos cinco nuevos registros de peces para el área marina del Parque Nacional Sistema Arrecifal Veracruzano, con evidencia fotográfica y se proveen los datos morfométricos y merísticos básicos: el miraestrellas Gillellus greyae (70.3 mm, Longitud total), el góbido Oxyurichthys stigmalophius (36 $\mathrm{mm}$, Longitud total), el cochito manchado Canthidermis maculata (342.4 mm, Longitud total) y el pez vela Istiophorus platypterus $(\approx 1,800 \mathrm{~mm}$, Longitud total) se distribuyen ampliamente en el Caribe; además del primer registro en el Golfo de México del lenguado manchado Bothus maculiferus (250 mm, Longitud total).

Palabras clave: Balistidae, Dactyloscopidae, Gobiidae, Istiophoridae, Isla de Enmedio, peces arrecifales.

\section{ABSTRACT}

We present four new fish records for the marine area of the Veracruzano Reef System National Park, records with photographic evidence, basic morphometric and meristic data are provided: the Arrow stargazer, Gillellus greyae (70.3 mm, Total length), the gobiid Oxyurichthys stigmalophius (36.6 mm, Total length), Rough triggerfish Canthidermis maculata (342.4 mm, Total length) and the Sailfish Istiophorus platypterus $(\approx 1,800 \mathrm{~mm}$, Total Length) with wide distribution in the Caribbean; in addition the first record in the Gulf of Mexico of the Mottled flounder Bothus maculiferus (250 mm, Total length).

Keywords: Balistidae, Dactyloscopidae, Gobiidae, Istiophoridae, Enmedio Island, reef fishes.

El área del Parque Nacional Sistema Arrecifal Veracruzano (PNSAV), comprende un polígono entre los $19^{\circ} 00^{\prime} 00^{\prime \prime}$ y $19^{\circ} 16^{\prime} 00^{\prime \prime} \mathrm{N}, 95^{\circ} 45^{\prime} 00^{\prime \prime}$ y $96^{\circ} 12^{\prime} 00^{\prime \prime} \mathrm{O}$, se localiza en la porción centro sur del Golfo de México, frente al estado de Veracruz (SEMARNAT, 2017; Tunnell et al., 2007), (Fig. 1). Representa al sistema arrecifal más grande en la región central del Golfo de México (Tunnell, 1992; Spalding et al., 2001) y después del Caribe mexicano, esta área natural protegida constituye el segundo complejo arrecifal más importante en la zona económica exclusiva del territorio nacional (Winfield et al., 2010). Está conformado por seis islas, diversos cayos y 27 arrecifes, cuatro de ellos son marginales y el resto de plataforma; de estos, 19 son emergidos y cuatro sumergidos (Ortiz-Lozano et al., 2013). 
El PNSAV es el sistema arrecifal mejor estudiado en el suroeste del Golfo de México (Jiménez-Hernández et al., 2007) y cuenta con un elevado número de especies. En el área se han registrado 472 especies de peces, incluyendo a dos especies invasoras (Del Moral-Flores et al., 2013; Robertson et al., 2016; Tello-Musi et al., 2018; Robertson et al., 2019). Sin embargo, con base en muestreos periódicos se han evidenciado nuevos registros ícticos para la zona, incrementando su diversidad faunística. Es por ello, que el presente trabajo tiene el objetivo registrar la presencia de cinco especies de actinopterigios en el área del PNSAV como resultado de una serie de monitoreos de la ictiofauna arrecifal en el área, durante marzo 2017 a febrero de 2019. Para la captura de los ejemplares pequeños se empleó una red de cuchara, mientras los ejemplares de mayor talla fueron obtenidos como parte de la pesca artesanal que se práctica en el área.

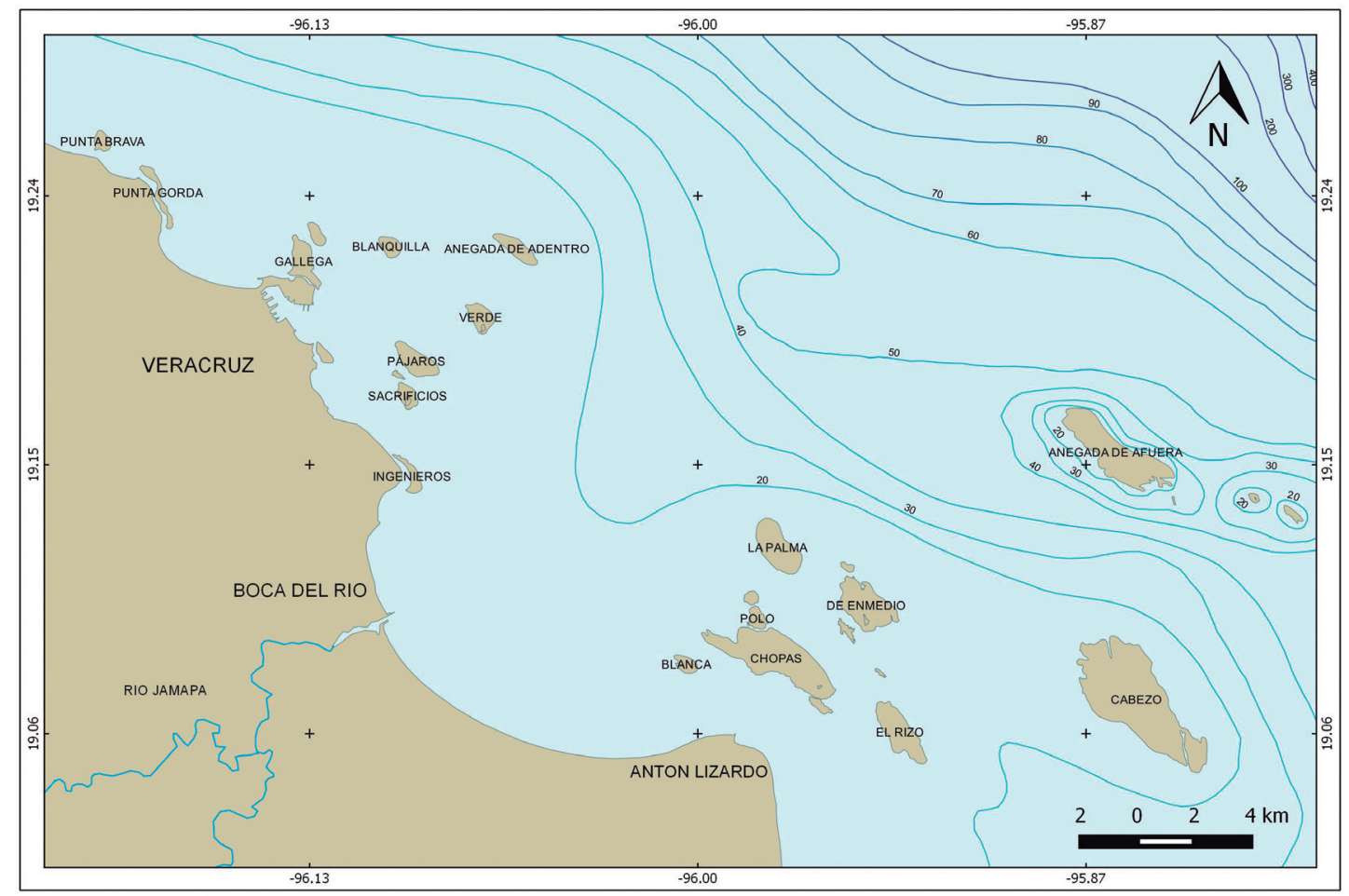

Figura 1. Parque Nacional Sistema Arrecifal Veracruzano (PNSAV), Golfo de México, México (elaboración propia).

Los organismos fueron fijados con formaldehido al $10 \%$ y preservados en alcohol etílico (70 \%), se depositaron en la Colección Ictiológica de la Facultad de Estudios Superiores Iztacala (CIFI) de la Universidad Nacional Autónoma de México. Se obtuvieron sus datos morfométricos y merísticos básicos. Se corroboró la identidad a nivel específico con ayuda de claves especializadas (Munroe, 2003; Nakamura, 2003; Williams, 2003; McEachran y Fechhelm, 2005) y de la descripción original y revisiones taxonómicas de los grupos (Gilbert, 1890; Kanazawa, 1952; Dawson, 1977, 1982; Mead y Böhlke, 1958; Pezold y Larson, 2015). El nombre científico actual de las especies fue validado mediante el trabajo de Fricke et al. (2020), mientras que el orden sistemático presentado sigue la propuesta de Van der Laan et al. (2020). 


\section{CLASE ACTINOPTERYGII \\ Orden Gobiiformes \\ Familia Gobiidae \\ Oxyurichthys stigmalophius (Mead \& Böhlke, 1958; Fig. 2A)}

Observaciones: 1 espécimen, CIFI- 538, colectado el 18 de marzo de 2017, por F. Del Moral, en barlovento de la planicie arrecifal de Isla de Enmedio, Veracruz. Morfometría: longitud total $36.6 \mathrm{~mm}$; longitud patrón $29 \mathrm{~mm}$; longitud cefálica $8.2 \mathrm{~mm}$; diámetro ocular $2.2 \mathrm{~mm}$; longitud del hocico $3.1 \mathrm{~mm}$; longitud predorsal $10.1 \mathrm{~mm}$; longitud preanal $15.5 \mathrm{~mm}$; altura corporal 5.3 mm. Merística: D: VI, 12; A: 13; C: 13; P1: 21; escamas con poro en la línea lateral 95.

El género Oxyurichthys se caracteriza por tener la tercera espina neural distalmente bífida o espatulada, el desarrollo de los canales cefálicos laterales se encuentra limitado a los canales óculo-escapulares con un patrón A'BCDFH'; no existen canales preoperculares o canales disyuntos por encima del opérculo. Esta conformación particular de los laterales cefálicos es única entre todos los peces gobioideos (Pezold, 1993). El género se encuentra representado por 20 especies, de las cuales $O$. stigmalophius es la única que se encuentra en el Atlántico occidental, desde Florida hasta Brasil, incluyendo a las Bahamas y Surinam. En el territorio mexicano solo se había observado en el sur del Golfo de México (Golfo de Campeche) (Mead y Böhlke, 1958; Pezold y Larson, 2015). Oxyurichthys stigmalophius presenta dos aletas dorsales, la primera con seis espinas, se origina de tras del margen posterior del opérculo, la segunda está constituida por una espina 13 elementos al igual que la aleta anal; 21 elementos en la aleta pectoral, aproximadamente 95 escamas en la serie longitudinal, cuatro manchas oscuras a lo largo del cuerpo, una mancha oscura en la región posterior de la primera aleta dorsal (Mead y Böhlke, 1958; Pezold y Larson, 2015).

\section{Orden Istiophoriformes \\ Familia Istiophoridae Istiophorus platypterus (Shaw, 1792; Fig. 2F-G)}

Observaciones: 1 espécimen, capturado de manera incidental el 12 de febrero de 2019, por pescador local, al sur del arrecife Cabezo, Veracruz. No se preservo debido al gran tamaño del ejemplar, de aproximadamente $1800 \mathrm{~mm}$ de longitud total.

Las especies de la familia Istiophoridae se reconocen por tener un hocico prolongado, que en una sección transversal es circular; tienen dos aletas anales y las aletas pélvicas son alargadas, de forma filamentosa; y dos quillas en la base de la cauda (Nakamura, 2003). En el Atlántico occidental se han registrado 5 especies de la familia. De ellas, Istiophorus platypterus tiene una coloración en azul oscuro en el dorso, la parte ventral es blanquecina y en los costados es pleteada; existen 19 a 20 barras verticales tenues en los costados. El espécimen se reconoció por tener la primera aleta dorsal bien desarrollada, los radios medios de mayor longitud que los primeros; los radios de las aletas pélvicas eran largos y al plegarse al cuerpo alcanzaban el orificio anal. Es necesaria revisar la sistemática del grupo, Nakamura (1983) reconoció a la población del Atlántico como Istiophorus albicans, que es menos robusta que la población del Indo-Pacífico.

\section{Orden Pleuronectiformes}

Familia Bothidae

Bothus maculiferus (Poey, 1860; Fig. 2E)

Observaciones: 1 espécimen, CIFI- 16, colectado el 14 de abril de 2012, por F. Del Moral, en la planicie arrecifal de Isla de Enmedio, Veracruz. Morfometría: longitud total $250 \mathrm{~mm}$; longitud patrón $210 \mathrm{~mm}$; longitud cefálica $54.1 \mathrm{~mm}$; diámetro ocular $12.5 \mathrm{~mm}$; longitud del 
hocico $12.5 \mathrm{~mm}$; longitud preanal $37.4 \mathrm{~mm}$; altura máxima $110 \mathrm{~mm}$. Merística: D: 92; A: 69; C: 14; P1: 11; escamas con poro en la línea lateral 113.

La familia Bothidae se identificó como sinistral con el ojo derecho en la porción dorsal superior del cráneo, las láminas olfativas estaban dispuestas transversalmente o irradiadas desde un raquis central. La familia comprende aproximadamente 20 géneros y 173 especies validas (Munroe, 2003; Fricke et al., 2020). Bothus maculiferus presenta la siguiente combinación de caracteres: margen anterior de la cabeza, convexo sin muesca por encima del maxilar, dos manchas oscuras difusas a lo largo del margen de la línea lateral en el lado ocular, no hay pigmentaciones distintas en los radios de la aleta dorsal o anal y presenta cuatro manchas localizadas en la aleta dorsal y anal. El número de branquiespinas en la rama inferior del primer arco branquial fue de 7 y la altura máxima corresponde al $52 \%$ de la longitud patrón (Munroe, 2003; Lara, 2006; Emery, 2016).

\section{Orden Blenniformes}

Familia Dactyloscopidae

Gillellus greyae Kanazawa, 1952 (Fig. 2 B, C)

Observaciones: 1 espécimen, CIFI- 537, colectado el 18 de marzo de 2017, por T. Hernández y E. Segovia, en la planicie arrecifal de Isla de Enmedio, Veracruz. Morfometría: longitud total $70.3 \mathrm{~mm}$; longitud patrón $61 \mathrm{~mm}$; longitud cefálica $14.8 \mathrm{~mm}$; diámetro ocular $2.1 \mathrm{~mm}$; longitud del hocico $4.8 \mathrm{~mm}$; longitud predorsal $11.7 \mathrm{~mm}$; longitud preanal $20.2 \mathrm{~mm}$. Merística: D: III-XVI, 21; A: II, 32; C: 8; P1: 12; P2: 3; escamas con poro en la línea lateral 54; 12 fimbrias inferiores.

La familia Dactyloscopidae habita en el medio marino, ocasionalmente invade los ambientes estuarinos (Castro-Aguirre et al., 1999). Su diversidad específica está representada por 48 especies validas, agrupadas en nueve géneros (Lin y Hastings, 2013; Fricke et al., 2020). Es una familia endémica de América; la mayor diversidad se encuentra en el Pacífico oriental, con 23 especies, mientras en el Atlántico occidental hay 19 especies (Nelson et al., 2016). El género Gillellus, conocidos como miraestrellas se caracterizan por poseer una aleta dorsal dividida que se origina en la nuca, la primera región está compuesta por tres espinas; fimbrias presentes en la región opercular y en labio inferior, obsoletas en el labio superior; presencia de nostrilos; boca moderadamente oblicua, la mandíbula inferior redondeada; ojo ligeramente protusible o en forma de cirro; modalmente de 12 a 13 radios de la aleta pectoral (Gilbert, 1890; Dawson, 1977, 1982).

El género Gillellus está representado por 10 especies validas (Fricke et al., 2020), de las cuales G. greyae, o también conocido como Miraestrellas flechas, se distingue por las siguientes características: dos aletas dorsales, la primera se origina en la nuca y la segunda está constituida por más de 17 espinas; más de 31 radios en la aleta anal; primer canal preopercular sin ramificar y con un solo poro distal; ausencia de fimbrias dermales en el labio superior y más de cuatro en el labio inferior; la mandíbula inferior proyectada más allá del margen posterior de la órbita ocular; escamas en la línea lateral, modalmente 52-57 (Kanazawa, 1952; McEachran y Fechhelm, 2005).

\section{Orden Tetraodontiformes}

Familia Balistidae

Canthidermis maculata (Bloch, 1786) (Fig. 2D)

Observaciones: 1 espécimen, CIFI-1596, colectado en marzo de 2018, por R. Ramón, en la zona costera oriental del arrecife Cabezo, PNSAV. Morfometría: longitud total 342.4 $\mathrm{mm}$; longitud patrón $279.1 \mathrm{~mm}$; longitud cefálica $87.5 \mathrm{~mm}$; diámetro ocular $15.6 \mathrm{~mm}$; 
longitud del hocico $53.8 \mathrm{~mm}$; longitud predorsal $93.6 \mathrm{~mm}$; longitud preanal $179.2 \mathrm{~mm}$; altura máxima $103.88 \mathrm{~mm}$. Merística: D: III, 25; A: 22; C: 10; P1: 13; escamas con poro en la línea lateral 55.

La familia Balistidae comprende 42 especies en 12 géneros, entre ellos el género Canthidermis con 3 especies: C. macrolepis, C. sufflamen, y C. maculata. Esta última con una distribución es circunglobal en mares tropicales (Matsuura, 2015; Nelson et al., 2016). El género se caracteriza por presentar una abertura nasal anterior en forma de embudo. Escamas en la mejilla con forma cuadrada en la parte anterior, en la parte posterior romboidales, con algunas escamas triangulares dispuestas horizontalmente con filas carnosas en medio. Escamas del cuerpo con crestas y una cresta grande en el medio anterior. Pedúnculo caudal, más largo que profundo, lateralmente elíptico, 8-10 hileras de escamas con crestas romas en el centro, dispuestas horizontalmente. Solapa ventral ausente y aleta caudal doble lunada (Sahayak et al., 2014).

La especie Gillellus greyae y Oxyurichthys stigmalophius se distribuyen ampliamente en el Caribe, y recientemente se registró en la zona a Canthidermis maculata. Sin embargo, no se reportaba su presencia en las costas de Veracruz. En la presente contribución se confirma su presencia y se amplía el área de distribución de estas especies en la región suroccidental del Golfo de México, mediante un ejemplar para cada especie. Para el caso de Bothus maculiferus, su distribución corresponde desde el Mar Caribe hasta el norte de Brasil (Emery, 2016), confirmado por primera vez su presencia en el Golfo de México. Conforme el esfuerzo de monitoreo aumente, permitirá un mayor conocimiento tanto temporal y regional de la ictiofauna del PNSAV.
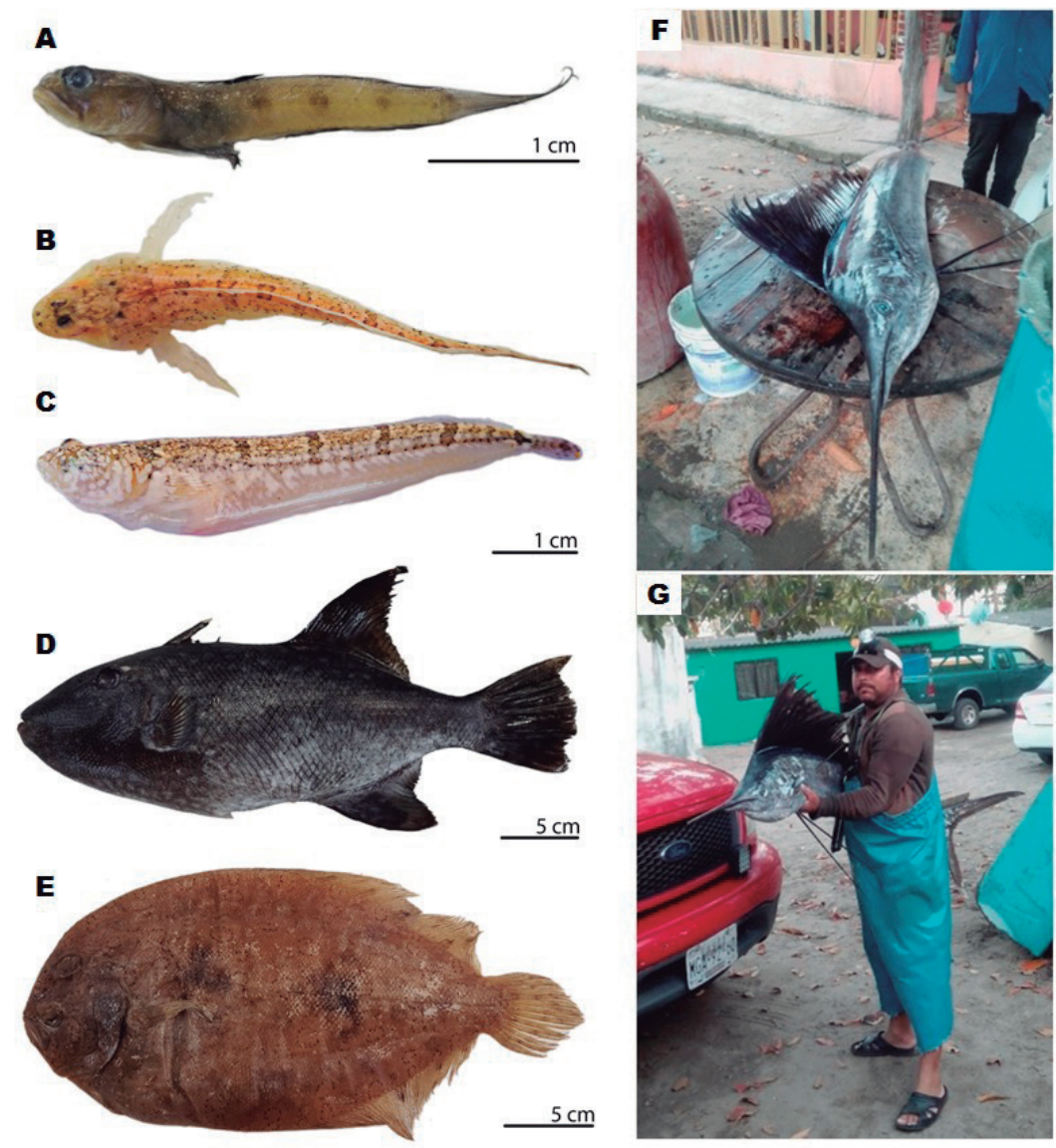

Figura 2. Ejemplares capturados en el PNSAV. A) Oxyurichthys stigmalophius, B) Gillellus greyae, vista dorsal, C) Gillellus greyae, vista lateral, D) Canthidermis maculata, E) Bothus maculiferus, F-G) Istiophorus platypterus (G, sostenido por Pedro Román Reyes). 


\section{AGRADECIMIENTOS}

Los autores agradecen a los compañeros que participaron en el trabajo de campo (B. González, S. Paleo, M. Medina, N. Ledesma, K. Guzmán, F. Guzmán y J. Rodríguez). A los pescadores de la comunidad de Antón Lizardo y Las Barrancas por el apoyo durante las fases de trabajo en campo. El primer autor agradece el apoyo al SIN-CONACYT, el segundo autor agradece al Posgrado en Ciencias del Mar y Limnología, UNAM.

\section{LITERATURA CITADA}

Castro-Aguirre, J. L., H. S. Espinosa y J. J. Schmitter-Soto. 1999. Ictiofauna estuarino-lagunar y vicaria de México. Limusa, Instituto Politécnico Nacional. México, 711 pp.

Dawson, C. E. 1977. Studies on eastern Pacific sand stargazers (Pisces: Dactyloscopidae). 4. Gillellus, Sindoscopus new genus, and Heteristius with description of new species. Proceedings of the California Academy of Sciences (Series 4), 41 (2): 125-160.

Dawson, C. E. 1982. Atlantic sand stargazers (Pisces: Dactyloscopidae), with description of one new genus and seven new species. Bulletin of Marine Science, 32 (1): 14-85.

Del Moral-Flores, L. F, J. L. Tello-Musi, H. Reyes-Bonilla, H. Pérez-España, J. A. Martínez-Pérez, G. Horta-Puga, L. A. Velazco-Mendoza y P. A. Álvarez del Castillo-Cárdenas. 2013. Lista sistemática y afinidades zoogeográficas de la ictiofauna del Sistema Arrecifal Veracruzano, México. Revista Mexicana de Biodiversidad, 84 (3): 825-846.

Emery, M. K. 2016. Taxonomic revisión of the genus Bothus (Bothidae; Pleuronectiformes). M. Sc. Thesis. University of Ottawa, Ottawa, Canada, 299 pp.

Fricke, R., W. N. Eschmeyer y R. Van der Laan (eds.). 2020. Echmeyer's catalogo of fishes: genera, species, references. Disponible en: http://researcharchive.calacademy.org/research/ ichthyology/catalog/fishcatmain.asp (accesado: 26 de marzo, 2020).

Gilbert, C. H. 1890. A preliminary report on the fishes collected by the steamer Albatross on the Pacific coast of North America during the year 1889, with descriptions of twelve new genera and ninety-two new species. Proceedings of the United States National Museum, 13 (797): 49-126.

Jiménez-Hernández, M. A., A. Granados-Barba y L. Ortiz-Lozano. 2007. Análisis de la información científica en el Sistema Arrecifal Veracruzano. En: Granados-Barba, A., A. L. G. Abarca y J. M. Vargas-Hernández (eds.). Investigaciones Cientificas en el Sistema Arrecifal Veracruzano. Universidad Autónoma de Campeche, Campeche, México, $1-16 \mathrm{pp}$.

Kanazawa, R. H. 1952. More new species and new records of fishes from Bermuda. Fieldiana Zoology, 34 (7): 71-100.

Lara, M. R. 2006. Bothidae: Lefteye Flounders. En: Richards, W. J. (ed.). Early Stages of Atlantic Fishes: An Identification Guide of Western Central North Atlantic. Taylor and Francis Group, CRC Press. Vol. 2: 2327-2343 pp.

Lin, H. C. y P. A. Hastings. 2013. Phylogeny and biogeography of a shallow water fish clade (Teleostei: Blenniiformes). BMC Evolutionary Biology, 13 (210): 1-19. 
Matsuura, K. 2015. Taxonomy and systematics of tetraodontiform fishes: a review focusing primarily on progress in the period from 1980 to 2014. Ichthyological Research, 62 (1): 72-113.

McEachran, J. D. y J. D. Fechhelm. 2005. Fishes of the Gulfof Mexico. Volume 2: Scorpaeniformes to Tetraodontiformes. University of Texas Press, Austin, 1004 pp.

Mead, G. W. y J. E. Böhlke. 1958. Gobionellus stigmalophius, a new goby from the Gulf of Campeche and the Great Bahama Bank. Copeia, 1958: 285-289.

Munroe, T. A. 2003. Order Pleuronectiformes; Bothidae. En: K. E. Carpenter (Ed.). The living marine resources of the Western Central Atlantic. Volume 3: Bony fishes part 2 (Opistognathidae to Molidae). FAO species identification guide for fishery purposes and American Society of Ichthyologist and Herpetologists Special Publication No. 5. FAO, Rome. v. 3: 1885-1895.

Nakamura, I. 1983. Systematics of the billfishes (Xiphiidae and Istiophoridae). Publications of the Seto Marine Biological Laboratory, 28 (5/6): 255-396.

Nakamura, I. 2003. Istiophoridae. Billfishes (spearfishes, marlins, and sailfishes). En: K. E. Carpenter (Ed.). The living marine resources of the Western Central Atlantic. Volume 3: Bony fishes part 2 (Opistognathidae to Molidae). FAO species identification guide for fishery purposes and American Society of Ichthyologist and Herpetologists Special Publication No. 5. FAO, Rome. v. 3: 1860-1866.

Nelson J. S., T. C. Grande y M. V. H. Wilson. 2016. Fishes of the World. 5 Ed. John Wiley \& Sons, Inc., Hoboken, New Jersey, 752 pp.

Ortiz-Lozano L, H. Pérez-España, A. Granados-Barba, C. González-Gándara, A. GutiérrezVelázquez y J. Martos. 2013. The Reef Corridor of Southwest Gulf of Mexico Challenge for its management and conservation. Ocean and Coastal Management, 86: 22-32.

Pezold, F. 1993. Evidence for a monophyletic Gobiinae. Copeia, 1993 (3): 634-643.

Pezold, F. L. y H. K. Larson. 2015. A revision of the fish genus Oxyurichthys (Gobioidei: Gobiidae) with descriptions of four new species. Zootaxa, 3988 (1): 1-95.

Robertson, D. R., N. Simoes, C. Gutiérrez-Rodríguez y V. J. Piñeros. 2016. An Indo-Pacific damselfish well established in the southern Gulf of Mexico: prospects for a wider, adverse invasion. Journal of the Ocean Science Foundation, 19: 1-17.

Robertson, D. R., H. Pérez-España, O. Domínguez-Domínguez, C. J. Estapé y A. M. Estapé. 2019. An update to the inventory of shore-fishes from the Parque Nacional Sistema Arrecifal Veracruzano, Veracruz, México. ZooKeys, 882: 127-157.

Sahayak, S., K. K. Joshi y V. Sriramachandramurty. 2014. Taxonomy of the Ocean Triggerfish, Canthidermis maculata (Tetradontiformes, Balistidae) from the Indian coast. Journal of the Marine Biological Association of India, 56: 56-61.

SEMARNAT (Secretaria de Medio Ambiente y Recursos Naturales). 2017. Programa de Manejo Parque Nacional Sistema Arrecifal Veracruzano. Comisión Nacional de Áreas Naturales Protegidas. 1 ed. México, 343 pp. 
Spalding, M., C. Ravilious y E. P. Green. 2001. World Atlas of Coral Reefs. Prepared at the UNEP World Conservation Monitoring Center, University of California Press, Berkeley, $\mathrm{CA}, 424 \mathrm{pp}$.

Tello-Musi, J. L., M. Chávez-Artega, F. Cruz-López y J. A. Martínez-Pérez. 2018. Adenda a la lista sistemática y afinidades zoogeográficas de la ictiofauna del Sistema Arrecifal Veracruzano, México. Revista de Zoología, 29: 81-83.

Tunnell, J. W. 1992. Natural versus human impacts to Southern Gulf of Mexico. Coral Reef Resources. Proceedings of the 7th International Coral Reef Symposium, Guam, Microneasia, 300-306 pp.

Tunnell, J. W., E. A. Chávez y K. Wither. 2007. Coral reefs of the southern gulf of Mexico. Texas A\&M University Press College Station, Station. 256 p.

Van der Laan, R., R. Fricke y W. N. Eschmeyer (Eds). 2020. Eschmeyer's catalog of fishes: classification.Disponible en: http:/www.calacademy.org/scientists/catalog-of-fishes-classification/ (accesado: 26 de marzo, 2020).

Williams, J. T. 2003. Dactyloscopidae. Pp. 1750-1753. In: K. E. Carpenter(Ed.). The living marine resources of the Western Central Atlantic. Volume 3: Bony fishes part 2 (Opistognathidae to Molidae). FAO species identification guide for fishery purposes and American Society of Ichthyologist and Herpetologists. Special Publication No. 5. FAO, Rome. v. 3: 1375-2127.

Winfield, I., S. Cházaro-Olvera, G. Horta-Puga, M. A. Lozano-Aburto y V. Arenas-Fuentes. 2010. Macrocrustáceos incrustantes en el Parque Nacional Sistema Arrecifal Veracruzano: biodiversidad, abundancia y distribución. Revista Mexicana de Biodiversidad, 81 (Supl): 165-175.

[Recibido: 26 de octubre, 2019. Aceptado para publicación: 06 de marzo, 2020] 